\title{
Step-index Si-Ge-core silica-cladded optical fibers
}

Mustafa Ordu ${ }^{1,2}$, Ahmet E. Akosman ${ }^{3}$, Jicheng Guo ${ }^{4,5}$, Shyamsunder Erramilli ${ }^{4,6,7}$, Siddharth Ramachandran ${ }^{4,7,8}$ and Soumendra N. Basu ${ }^{1,4,7}$ ${ }^{I}$ Department of Mechanical Engineering, Boston University, Boston, USA

${ }^{2}$ UNAM - National Nanotechnology Research Center and Institute of Materials Science and Nanotechnology Bilkent University, Ankara, 06800, Turkey.

${ }^{3}$ School of Engineering, Roger Williams University, Bristol, RI 02809, USA.

${ }^{4}$ Division of Materials Science and Engineering, Boston University, Brookline, MA 02446, USA.

${ }^{5}$ Argonne National Laboratory, Lemont, IL 60439, USA.

${ }^{6}$ Department of Physics, Boston University, Boston, MA 02215, USA.

${ }^{7}$ Photonics Center, Boston University, Boston, MA 02215, USA

${ }^{8}$ Department of Electrical and Computer Engineering, Boston University, Boston, MA 02215, USA.

ordu@unam.bilkent.edu.tr,aakosman@rwu.edu

Abstract-Si-Ge alloy-core silica-cladded fibers were drawn at a low temperature to minimize impurity diffusion. The elemental segregation in the as-drawn fibers was overcome by a thermal treatment. The transmission losses of the fibers were calculated as $28 \mathrm{~dB} / \mathrm{cm}$ at $6.1 \mu \mathrm{m}$ wavelength.

Keywords—Si-Ge alloys, mid-IR optics, semiconductor fibers

\section{INTRODUCTION}

The usage of semiconductor-core glass-cladded optical fibers for mid-infrared (IR) transmission has been a subject of several studies due to applications such as remote laser delivery and chemical sensing [1]. A silicon-core fiber was proposed for the first time by using the high-pressure chemical vapor deposition technique [2]. Over the years, unary and binary semiconductors have been proposed for the fiber core material by combining different semiconductor cores and glass claddings $[3,4]$. Binary semiconductor-cores such as $\mathrm{Si}-$ Ge have advantages over the unary-core fibers, allowing tuning of refractive indices and transmission windows. In several studies, Si-Ge fibers have been fabricated by the rodin-tube method, in which semiconductors are placed in glass tubes and are drawn in a draw tower $[4,5]$. However, the asdrawn fibers have nonhomogeneous core structures resulting in high transmission losses. In this work, Si-Ge alloy-core silica-cladded fibers were drawn at a relatively low temperature to minimize impurity diffusion. The as-drawn fibers were then annealed to improve the homogeneity of the core, and Si-Ge step-index (SI) fibers were formed. Fibers were characterized in the mid-IR spectrum, and transmission loss of the annealed fibers was measured $28 \mathrm{~dB} / \mathrm{cm}$ at $6.1 \mu \mathrm{m}$. Numerical simulations are presented as possible pathways for obtaining low-loss mid-IR fibers.

\section{EXPERIMENTAL METHODS}

\section{A. Preform and Fiber drawing}

A core drilled Ge rod was placed inside a partially-drilled $\mathrm{Si}$ rod that had itself been core-drilled. Both the Ge rod and $\mathrm{Si}$ piece were machined out of $99.999 \%$ purity monocrystalline blocks. The core material was placed in a silica-glass tube with $3 \mathrm{~mm}$ inner diameter and $9 \mathrm{~mm}$ outer diameter. Additional silica tubes can be added in order to adjust the core/cladding diameter ratio [3]. The rod-in-tube method was chosen due to its capability of fabricating long fibers. Fibers were drawn with a laboratory-made draw tower at $1760^{\circ} \mathrm{C}$.

\section{B. Thermal annealing}

The compositional improvement of the fiber cores was achieved by annealing the fibers in a box furnace under the normal atmospheric conditions. Different strategies were applied to obtain the highest uniformity of the fiber core [4], and the annealing recipe in Table 1 resulted in uniform SI SiGe alloy-cores.

TABLE I. THE RECIPE OF THE THERMAL TREATMENT FOR UNIFORM CORE STRUCTURES (R.T. : ROOM TEPERATURE).

\begin{tabular}{|c|c|c|c|}
\hline Action & T $\left({ }^{\circ} \mathbf{C}\right)$ & Rate $\left({ }^{\circ} \mathbf{C} / \mathbf{m i n}\right)$ & Time (min) \\
\hline Starting & R.T. & - & - \\
\hline Heating & 1465 & 8 & 180 \\
\hline Dwelling & 1465 & - & 6 \\
\hline Cooling & 1350 & 15 & 8 \\
\hline Dwelling & 1350 & - & 6 \\
\hline Cooling & 900 & 2 & 225 \\
\hline Cooling & R.T. & Air-cooling & $\sim 200$ \\
\hline
\end{tabular}

\section{RESULTS}

\section{A. Microstructure of the fibers}

The microstructures of the as-drawn and annealed fibers were observed by scanning electron microscope (SEM) based energy dispersive x-ray (EDX) technique. Fibers were polished in cross-section using standard polishing methods.

The cross-sectional images of the as-drawn and annealed fibers are shown in Figure 1(a) and 1(b), respectively. The fibers core diameter is $27 \mu \mathrm{m}$ and the diameter of the cladding is $245 \mu \mathrm{m}$. Random nucleation of the $\mathrm{Si}$ and Ge during solidification resulted in a nonhomogeneous core. The uniformity of the core after the annealing with the recipe at Table 1 points the effectiveness of the annealing method.

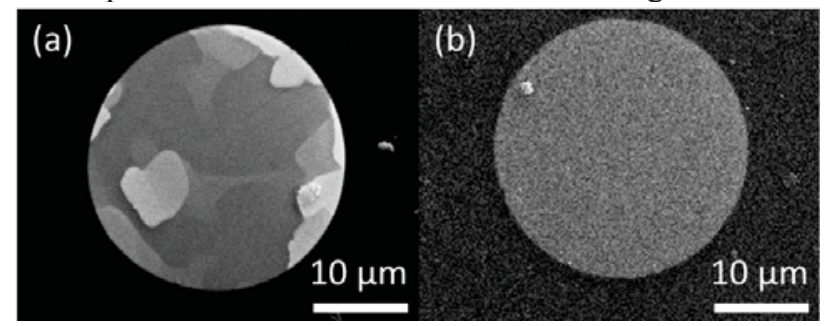

Fig. 1. Cross-sectional view of the fiber (a) before and (b) after the thermal treatment. Dark outer parts are the glass claddings.

Figure 2 shows the EDX dot mapping of the thermally treated fiber for $\mathrm{Si}, \mathrm{Ge}$ and $\mathrm{O}$, supporting the increased uniformity of the core. The atomic weight percentage of the Si-Ge alloy-core was calculated as $87.5 \% \mathrm{Si}, 9.5 \% \mathrm{Ge}$ and $3 \%$ $\mathrm{O}$. The amount of oxygen stayed same before and after the thermal treatment, proving that the post-drawing process did not cause further diffusion from the cladding to the core.

\section{B. Optical characterization}

Fibers were characterized by a quantum cascade laser (QCL) at $6.1 \mu \mathrm{m}$ for a reliable measurement. A ZnSe objective 
with $6 \mathrm{~mm}$ focal length was used for the input coupling and two $\mathrm{ZnSe}$ convex lenses with $25.4 \mathrm{~mm}$ focal length were used to collect the fiber output. The output light was later guided to a mercury cadmium telluride (MCT) detector to measure the transmission.

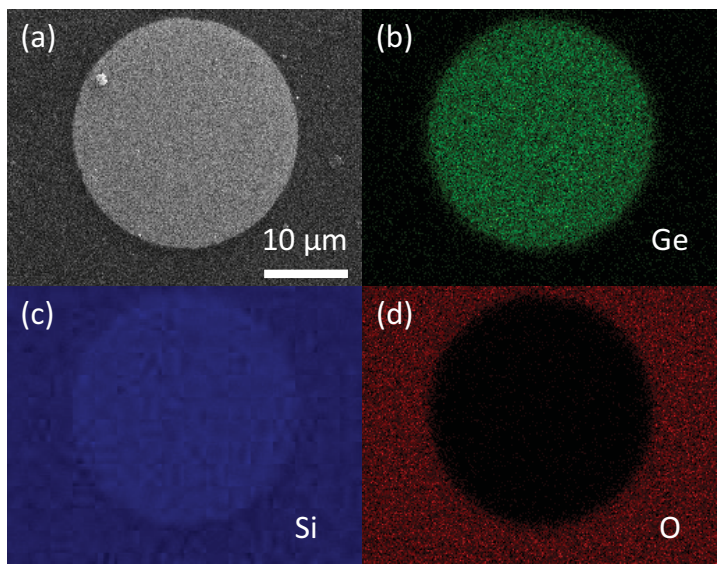

Fig. 2. (a) SEM image of the fiber cross-section after the thermal treatment and the SEM-EDX dot mappinf of (b) Ge, (c) Si and (d) O.

The transmission losses of the fiber were calculated by using the standard cutback method with three fiber length: $7.90 \mathrm{~mm}, 6.60 \mathrm{~mm}$ and $5.42 \mathrm{~mm}$. The latter two-lengths were obtained by polishing the $7.90 \mathrm{~mm}$ fiber twice at the output end. The transmission loss of the fiber at $6.1 \mu \mathrm{m}$ was found 28 $\mathrm{dB} / \mathrm{cm}$. One reason behind the relatively high losses of the SiGe SI fiber could be due to the devitrification of the silica cladding during the post-drawing process. The devitrification of the silica accelerates above $1100^{\circ} \mathrm{C}$, forming micron-sized cracks at the cladding. The cracks close to the core can penetrate through and may increase the transmission losses.

\section{PATHWAYS TO LOW LOSS FIBERS}

In order to create ultimate low-loss semiconductor-core fibers, multiple strategies were computationally investigated. One way is to form graded-index (GRIN) structures at the core of the fibers. A second method is to include an IR transmitting layer between the semiconductor-core and the glass cladding. In principle, the layer can be selected from different materials such as soft-glasses or ceramics, as long as the layer transmits the spectrum and has a lower refractive index than the core. Such interlayers of chalcogenide glasses (AsSe or AsS) showed promising results. However, the working temperature range of chalcogenides and silica glasses (or even borosilicate glasses) are too apart from each other that it is practically impossible to draw fibers. Also, materials such as $\mathrm{GaN}$ and $\mathrm{Al}_{2} \mathrm{O}_{3}$ are good alternatives but the high melting points cause difficulties for silica glasses.

To investigate the impact of the GRIN structures and interlayers, first the confinement losses of a pure Ge core fiber and the proposed Si-Ge SI alloy-core were simulated with Finite Element Method. Then, a Ge core fiber surrounded by a Si layer as a double layer structure is built, which is the base form of the GRIN structure. Lastly, the confinement loss of a GRIN structure composed of several layers of different Si-Ge alloy compositions is numerically calculated.

Figure 3 shows the simulation results of the proposed fiber structures. The results indicates that the proposed $\mathrm{Si}-\mathrm{Ge}$ alloy core fiber exhibits a lower confinement loss compared to the Ge core fiber for the wavelengths longer than $5.4 \mu \mathrm{m}$. While the proposed alloy core fiber is promising for lower losses compared to bare core structure, further improvement is still needed. Therefore, the proposed double layer structure is investigated and shows a much better loss profile for the entire spectrum. The final GRIN structure shows an ultimate pathway for a uniform low loss performance, however the losses can be further decreased with more optimized geometrical parameters. Nonetheless, the proposed GRIN structure with $\mathrm{Si}-\mathrm{Ge}$ alloys is a promising candidate for uniform low loss optical fibers over long wavelength intervals. Low-loss semiconductor fibers have potential application in nonlinear optics owing to the high nonlinearities of semiconductors $[6,7]$.

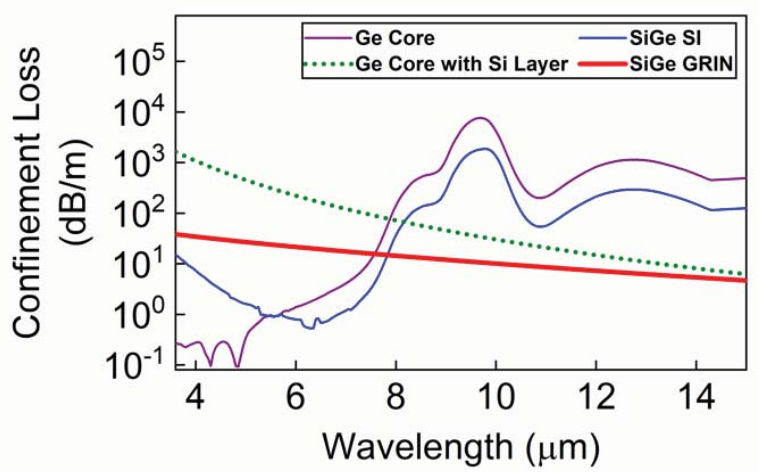

Fig. 3. Simulation results of Ge core, Ge core with Si layer, SiGe SI, and SiGe GRIN optical fibers. Both GRIN and additional Si layer improves the transmission properties of the semiconductor cores.

\section{CONCLUSIONS}

In this work, Si-Ge alloy-core silica-cladded fibers for midIR transmission were drawn and thermally treated. Annealed fibers were characterized with a QCL light source at $6.1 \mu \mathrm{m}$, and the transmission loss was found $28 \mathrm{~dB} / \mathrm{cm}$. Numerical studies suggest that low-loss fibers in wide transmission windows are possible by forming GRIN structure or by adding an IR transmitting layer between the semiconductor core and glass cladding. Future work will concentrate on the fabrication of these fiber structures.

\section{ACKNOWLEDGMENTS}

This project has been supported by the National Science Foundation (NSF, Grand Number CMMI-1301108, 2013).

\section{REFERENCES}

[1] G. Tao et al., "Infrared fibers," Advances in Optics and Photonics, vol. 7, no. 2, pp. 379-458, 2015.

[2] P. J. Sazio et al., "Microstructured optical fibers as high-pressure microfluidic reactors," Science, vol. 311, no. 5767, pp. 1583-1586, 2006.

[3] M. Ordu et al., "Mid-infrared transmission through germanium-core borosilicate glass-clad semiconductor fibers," Optical Materials Express, vol. 7, no. 9, pp. 3107-3115, 2017.

[4] M. Ordu, et al., "Effect of Thermal Annealing on Mid-Infrared Transmission in Semiconductor Alloy-Core Glass-Cladded Fibers," Advanced Fiber Materials, pp. 1-7, 2020.

[5] D. A. Coucheron et al., "Laser recrystallization and inscription of compositional microstructures in crystalline SiGe-core fibres," Nature communications, vol. 7, no. 1, pp. 1-9, 2016.

[6] M. Ordu et al., "Nonlinear optics in germanium mid-infrared fiber material: Detuning oscillations in femtosecond mid-infrared spectroscopy," AIP Advances, vol. 7, no. 9, p. 095125, 2017.

[7] A. C. Peacock et al., "Wavelength conversion and supercontinuum generation in silicon optical fibers," IEEE Journal of Selected Topics in Quantum Electronics, vol. 24, no. 3, pp. 1-9, 2017. 